PROCEEDINGS OF THE

AMERICAN MATHEMATICAL SOCIETY

Volume 139, Number 8, August 2011, Pages 2807-2820

S 0002-9939(2011)10842-6

Article electronically published on March 2, 2011

\title{
BOUNDARIES OF ESCAPING FATOU COMPONENTS
}

\author{
P. J. RIPPON AND G. M. STALLARD
}

(Communicated by Mario Bonk)

\begin{abstract}
Let $f$ be a transcendental entire function and $U$ be a Fatou component of $f$. We show that if $U$ is an escaping wandering domain of $f$, then most boundary points of $U$ (in the sense of harmonic measure) are also escaping. In the other direction we show that if enough boundary points of $U$ are escaping, then $U$ is an escaping Fatou component. Some applications of these results are given; for example, if $I(f)$ is the escaping set of $f$, then $I(f) \cup\{\infty\}$ is connected.
\end{abstract}

\section{INTRODUCTION}

Let $f$ be a transcendental entire function and denote by $f^{n}, n=0,1,2, \ldots$, the $n$th iterate of $f$. The Fatou set $F(f)$ is defined to be the set of points $z \in \mathbb{C}$ such that $\left(f^{n}\right)_{n \in \mathbb{N}}$ forms a normal family in some neighborhood of $z$. The components of $F(f)$ are called Fatou components. The complement of $F(f)$ is called the Julia set $J(f)$. An introduction to the properties of these sets can be found in 3 .

The set $F(f)$ is completely invariant, so for any component $U$ of $F(f)$ there exists, for each $n=0,1,2, \ldots$, a component of $F(f)$, which we call $U_{n}$, such that $f^{n}(U) \subset U_{n}$. If, for some $p \geq 1$, we have $U_{p}=U_{0}=U$, then we say that $U$ is a periodic component of period $p$, assuming $p$ to be minimal. There are then four possible types of periodic components; see [3, Theorem 6]. If $U_{n} \neq U_{m}$ for $m \neq n$, then we say that $U$ is a wandering domain of $f$.

The escaping set

$$
I(f)=\left\{z: f^{n}(z) \rightarrow \infty \text { as } n \rightarrow \infty\right\}
$$

was first studied for a general transcendental entire function $f$ by Eremenko 7 . He proved that

$$
I(f) \cap J(f) \neq \emptyset \quad \text { and } \quad \partial I(f)=J(f),
$$

and also that $\overline{I(f)}$ has no bounded components. Eremenko remarked that it is plausible that all the components of $I(f)$ are unbounded, a statement now known as Eremenko's conjecture that remains open in spite of much work on it and many partial results.

Any Fatou component that meets $I(f)$ must lie in $I(f)$ by normality; we call such components escaping Fatou components. Escaping wandering domains can

Received by the editors July 23, 2010.

2010 Mathematics Subject Classification. Primary 37F10; Secondary 30D05.

Both authors were supported by EPSRC grant EP/H006591/1.

(C)2011 American Mathematical Society 
be bounded or unbounded, and escaping periodic Fatou components are all unbounded; the latter are called Baker domains. This paper gives results about the relationship between an escaping Fatou component and its boundary and describes some consequences of these results.

For the function $f(z)=z+1+e^{-z}$, studied by Fatou in [8], the set $F(f)$ is a completely invariant Baker domain, whose boundary is $J(f)$. So in this example an escaping Fatou component has many boundary points that are not in $I(f)$. It is natural to ask whether every escaping Fatou component of a transcendental entire function must have at least one boundary point in $I(f)$. We have the following partial result.

Theorem 1.1. Let $f$ be a transcendental entire function and let $U$ be a wandering domain of $f$ such that $U \subset I(f)$. Then

$$
\partial U \cap I(f) \neq \emptyset .
$$

Moreover, the set $\partial U \cap I(f)^{c}$ has zero harmonic measure relative to $U$.

The proof of Theorem 1.1 can be adapted to show that $\partial U \cap I(f) \neq \emptyset$ for many Baker domains (see Section 2, Remark 2), but it remains open whether this conclusion holds whenever $U$ is a Baker domain.

In the other direction to Theorem 1.1, we can ask whether a Fatou component must be escaping if a large enough subset of its boundary is escaping. We have the following result in this direction; in particular this result implies that if $U$ is a Fatou component of $f$ such that $\partial U \subset I(f)$, then $U \subset I(f)$.

Theorem 1.2. Let $f$ be a transcendental entire function and let $U$ be a Fatou component of $f$.

(a) If $\partial U \cap I(f)$ has positive harmonic measure relative to $U$, then $U \subset I(f)$.

(b) If $\partial U \cap A(f)$ has positive harmonic measure relative to $U$, then $U \subset A(f)$.

The set $A(f)$ is the fast escaping set, introduced by Bergweiler and Hinkkanen in [5], which can be defined as follows (see [17]):

$$
A(f)=\left\{z: \text { there exists } L \in \mathbb{N} \text { such that }\left|f^{n+L}(z)\right| \geq M^{n}(R) \text {, for } n \in \mathbb{N}\right\} .
$$

Here $M(r)=M(r, f)=\max _{|z|=r}|f(z)|$ and $R>0$ is such that $M(r)>r$ for $r \geq R$ or equivalently such that $M^{n}(R) \rightarrow \infty$ as $n \rightarrow \infty$.

Many of the properties of $A(f)$ are stronger than those of $I(f)$ (see Section 4). For example, $A(f)$ has the following stronger property than that given for $I(f)$ in Theorem 1.1 (see [17, Theorem 1.2]):

$$
\text { if } U \text { is a Fatou component of } f \text { that meets } A(f) \text {, then } \bar{U} \subset A(f) \text {. }
$$

Note that it is possible for a Fatou component to lie in $A(f)$; for example,

$$
\text { if } U \text { is a multiply connected Fatou component of } f \text {, then } \bar{U} \subset A(f)
$$

(see [14]), and Bergweiler has constructed a transcendental entire function with both simply connected and multiply connected Fatou components in $A(f)$ (see [4]). All Fatou components in $A(f)$ are wandering domains (see [5]).

We prove Theorems 1.1 and 1.2 in Sections 2 and 3, respectively. In Section 4 we give two consequences of these theorems, one of which states that if $f$ is a transcendental entire function, then $I(f) \cup\{\infty\}$ is connected, and the other of which is an improved version of a result from [15] which gives a sufficient condition 
for $I(f)$ to be connected; the proof we give of the latter result was inspired by an unpublished idea of Professor Noel Baker. This section also includes a short proof that $I(f) \neq \emptyset$, which may be of independent interest.

Section 5 is concerned with the components of $I(f)$; for example, we give a new sufficient condition for the components of $I(f)$ to be unbounded and prove that various sets, such as $I(f)$ and $A(f)$, and their complements, are either connected or have infinitely many components.

\section{Proof of Theorem 1.1}

First we recall that for a domain $G$ and a set $E \subset \partial G$, the harmonic measure of $E$ at $z$ relative to $G$, denoted by $\omega(z, E, G)$, is the solution of the Dirichlet problem in $G$ (found by using the Perron method) with boundary values given by the characteristic function $\chi_{E}$. See [9] or [12, for example, for the solution of the Dirichlet problem and the properties of harmonic measure.

In any simply connected domain $G$ in $\mathbb{C}$, all points $\zeta \in \partial G$ are regular for the Dirichlet problem (see [12]); that is, if $\phi$ is any real-valued function on $\partial G$ which is continuous at $\zeta \in \partial G$ and $H_{\phi}$ is the solution of the Dirichlet problem in $G$ with boundary values $\phi$, then $H_{\phi}(z) \rightarrow \phi(\zeta)$ as $z \rightarrow \zeta$.

Proof of Theorem 1.1. Suppose that $U$ is an escaping wandering domain of $f$. Fix $z_{0} \in U$ and, for $n \geq 0$, let $z_{n}=f^{n}\left(z_{0}\right)$ and $U_{n}$ be the Fatou component that contains $f^{n}(U)$. In view of (1.3), we can assume that each $U_{n}$ is simply connected.

Let $R>0$ and put

$$
B_{n}=B_{n}(R)=\left\{z \in \partial U:\left|f^{n}(z)\right| \leq R\right\}, \quad n \in \mathbb{N} .
$$

To prove that $\partial U \cap I(f)^{c}$ has zero harmonic measure relative to $U$, it is sufficient to show that, for each $R>0$,

$$
\bigcap_{m \geq 1} \bigcup_{n \geq m} B_{n}=\left\{z \in \partial U:\left|f^{n}(z)\right| \leq R \text { for infinitely many } n\right\}
$$

has harmonic measure 0 relative to $U$.

Define $\Delta=\{z:|z|>R\} \cup\{\infty\}$, considered as a disc in the Riemann sphere $\hat{\mathbb{C}}$, and choose $N=N(R)$ such that $\left|z_{n}\right|>2 R$ for $n \geq N$. Then, for $n \geq N$, define

$$
E_{n}=\partial U_{n} \cap\{z:|z| \leq R\},
$$

$V_{n}$ to be the component of $U_{n} \cap \Delta$ that contains $z_{n}$,

$$
F_{n}=\partial V_{n} \cap\{z:|z|=R\}
$$

see Figure 1, We have

$$
\omega\left(z, E_{n}, U_{n}\right) \leq \omega\left(z, F_{n}, \Delta\right), \quad \text { for } z \in V_{n}, n \geq N .
$$

This inequality is a special case of Carleman's domain extension principle, and it is proved as follows. The function

$$
u(z)= \begin{cases}0, & z \in \Delta \backslash V_{n}, \\ \omega\left(z, E_{n}, U_{n}\right), & z \in V_{n},\end{cases}
$$

is subharmonic in $\Delta$ because $\omega\left(z, E_{n}, U_{n}\right)$ has boundary value 0 at all points of $\partial V_{n} \cap \Delta$ (since $V_{n}$ is simply connected and hence regular for the Dirichlet problem). Also,

$$
\limsup _{z \rightarrow \zeta} u(z) \leq \begin{cases}0, & \text { for } \zeta \in \partial \Delta \backslash F_{n} \\ 1, & \text { for } \zeta \in F_{n}\end{cases}
$$




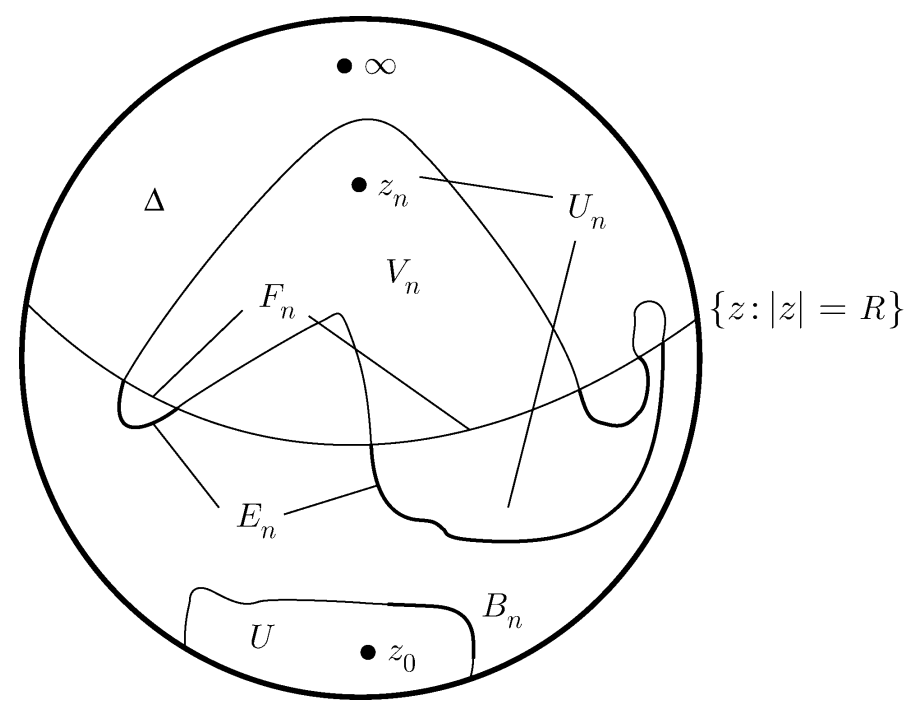

Figure 1. The sets $U, B_{n}, \Delta, U_{n}, V_{n}, E_{n}$ and $F_{n}$

because $F_{n}$ is a closed subset of $\partial \Delta$. So (2.2) holds by the maximum principle for subharmonic functions (see [12, Theorem 2.3.1]).

By conformally mapping $\Delta$ onto $\{z:|z|<1 / R\}$ and applying Harnack's inequality (see [12, Theorem 1.3.1]) in that disc, we obtain

$$
\omega\left(z_{n}, F_{n}, \Delta\right) \leq 3 \omega\left(\infty, F_{n}, \Delta\right), \quad \text { for } n \geq N,
$$

as $\left|z_{n}\right|>2 R$ for $n \geq N$. Since $U$ is a wandering domain, the sets $F_{n}$ are disjoint, so we deduce from (2.2) and (2.3) that

$$
\sum_{n \geq N} \omega\left(z_{n}, E_{n}, U_{n}\right) \leq 3 \sum_{n \geq N} \omega\left(\infty, F_{n}, \Delta\right) \leq 3 \omega(\infty, \partial \Delta, \Delta)=3 .
$$

Now $f^{n}(U) \subset U_{n}$ and $f^{n}\left(B_{n}\right) \subset E_{n}$, so

$$
\omega\left(z, B_{n}, U\right) \leq \omega\left(f^{n}(z), E_{n}, U_{n}\right), \quad \text { for } z \in U, n \geq N,
$$

by [12, Theorem 4.3.8]. In particular,

$$
\omega\left(z_{0}, B_{n}, U\right) \leq \omega\left(z_{n}, E_{n}, U_{n}\right), \quad \text { for } n \geq N .
$$

Hence, by (2.4),

$$
\sum_{n \geq N} \omega\left(z_{0}, B_{n}, U\right) \leq \sum_{n \geq N} \omega\left(z_{n}, E_{n}, U_{n}\right)<\infty
$$

so

$$
\omega\left(z_{0}, \bigcup_{n \geq m} B_{n}, U\right) \leq \sum_{n \geq m} \omega\left(z_{0}, B_{n}, U\right) \rightarrow 0 \quad \text { as } m \rightarrow \infty,
$$

which gives (2.1).

Remarks. 1. It is natural to ask if there is an example of an entire function with an escaping wandering domain that has at least one non-escaping boundary point.

2. The proof of Theorem 1.1 can be adapted to show that, for many Baker domains $U$, the set $\partial U \cap I(f)^{c}$ has zero harmonic measure relative to $U$. For 
example, suppose that $U$ is an invariant Baker domain in which there is an orbit $z_{n}=f^{n}\left(z_{0}\right), n \in \mathbb{N}$, such that

$$
\left|z_{n+1}\right| \geq k\left|z_{n}\right|, \quad \text { for } n \in \mathbb{N}
$$

where $k>1$. This is the case for several types of Baker domains; see [13, Section 2] for examples. Then, with the notation from the proof of Theorem 1.1 we have $U_{n}=U$ and $E_{n}=\partial U \cap\{z:|z| \leq R\}=E$, say, for $n \in \mathbb{N}$, and

$$
\omega(z, E, U) \leq \frac{C}{|z|^{1 / 2}}, \quad \text { for } z \in U \cap \Delta,
$$

where $C>0$ is an absolute constant. Since $U$ is simply connected, this last harmonic measure estimate can be obtained from the Beurling projection theorem (see 9. Theorem 9.2, page 105]), by exchanging the roles of 0 and $\infty$. By (2.6) and (2.7), we deduce that (2.5) holds, so the conclusion of Theorem 1.1 also holds in this situation.

\section{Proof of Theorem 1.2}

The proof of Theorem 1.2 uses the following version of the maximum principle for subharmonic functions (see [9, page 102] or [12, Theorem 3.6.9]).

Lemma 3.1. Let $G$ be a domain in $\mathbb{C}$ and let $u$ be a subharmonic function in $G$ which is bounded above. If $\partial G$ is not a polar set and

$$
\limsup _{z \rightarrow \zeta} u(z) \leq 0, \quad \text { for } \zeta \in \partial G \backslash E,
$$

where $E$ is a polar subset of $\partial G$, then $u \leq 0$ in $G$.

Polar sets are defined in [9] and [12; here we just need the facts that if a set contains a continuum, then it is not polar whereas a finite set is polar.

Proof of Theorem 1.2. In view of (1.3), we can assume throughout the proof that $U$ is simply connected; in particular, $\partial U$ is not a polar set and all points of $\partial U$ are regular for the Dirichlet problem.

Part (a). We consider two cases. First we suppose that $F(f)$ is disconnected. In this case we claim that there is a closed disc which lies outside $\bigcup_{n \geq 0} f^{n}(U)$. This is clearly true if $U$ is forwards invariant under $f$. If $U$ is not forwards invariant, then there is a Fatou component $V$ of $f$ different from $U$ such that $f(V) \subset U$. Moreover, we claim that we can choose $V$ to be different from all $f^{n}(U), n \geq 0$. For otherwise, $U$ is periodic under $f$, with period $p>1$, and the $p$ Fatou components of $f$ in the orbit of $U$ are each completely invariant under $f^{p}$. This contradicts a theorem of Baker [1] which states that a transcendental entire function can have at most one completely invariant Fatou component.

By making an affine change of variables, we can assume that $\{z:|z| \leq 1\}$ lies outside $\bigcup_{n \geq 0} f^{n}(U)$. Thus the functions

$$
u_{n}(z)=\log \left|f^{n}(z)\right|, \quad z \in \bar{U}, n \in \mathbb{N},
$$

are positive harmonic in $U$ and continuous in $\bar{U}$. By hypothesis there exists a set $E \subset \partial U$ such that the harmonic measure $\omega(z, E, U)>0$, for $z \in U$, and

$$
u_{n}(\zeta) \rightarrow \infty \text { as } n \rightarrow \infty, \quad \text { for } \zeta \in E \text {. }
$$


Since $\omega(z, ., U)$ is a positive finite Borel measure on $\partial U$, we can assume by Egorov's theorem (see [19], for example) that

$$
u_{n} \rightarrow \infty \text { as } n \rightarrow \infty \text {, uniformly on } E \text {, }
$$

and we can assume in addition that $E$ is closed. Thus if $C>0$ is given, then there exists $N=N(C)$ such that

$$
u_{n}(\zeta) \geq C, \quad \text { for } \zeta \in E, n \geq N .
$$

Now consider the bounded harmonic functions

$$
v_{n}(z)=C \omega(z, E, U)-u_{n}(z), \quad z \in U, n \geq N .
$$

Then, for $n \geq N$,

$$
\limsup _{z \rightarrow \zeta} v_{n}(z) \leq \begin{cases}C-u_{n}(\zeta) \leq 0, & \text { for } \zeta \in E, \\ \lim _{z \rightarrow \zeta} C \omega(z, E, U)-u_{n}(\zeta) \leq 0, & \text { for } \zeta \in \partial U \backslash E .\end{cases}
$$

The first inequality follows from (3.1) and the fact that $\omega(z, E, U) \leq 1$ for $z \in U$. The second inequality holds because $u_{n}(z)>0$ for $z \in U$ and $\lim _{z \rightarrow \zeta} \omega(z, E, U)=0$, for $\zeta \in \partial U \backslash E$, since all points of $\partial U$ are regular and $E$ is closed, and $\omega(z, E, U)$ is the solution of the Dirichlet problem in $U$ with boundary values $\chi_{E}$.

Also, in the case that $U$ is unbounded, the point at infinity acts as a polar set in the boundary of $U$ (see [12, p. 85 and Corollary 3.2.5]).

It follows by Lemma 3.1 that $v_{n}(z) \leq 0$, for $z \in U$. Hence

$$
u_{n}(z)=\log \left|f^{n}(z)\right| \geq C \omega(z, E, U), \quad \text { for } z \in U, n \geq N .
$$

Since $C>0$ is arbitrary, it follows that

$$
u_{n}(z)=\log \left|f^{n}(z)\right| \rightarrow \infty \text { as } n \rightarrow \infty, \quad \text { for } z \in U,
$$

so $U \subset I(f)$.

In the second case, we suppose that $U=F(f)$ is connected, from which it follows that $f^{n}(U) \subset U$, for $n \in \mathbb{N}$. In this case, $U$ is simply connected and unbounded, so we can define

$$
\psi(z)=k \sqrt{z-a}+b, \quad z \in U,
$$

where $a \in J(f), \psi$ is conformal on $U$, and $k>0$ and $b \in \mathbb{C}$ are chosen so that $\psi(U) \cap\{z:|z| \leq 1\}=\emptyset$. Note that for $z \in U$ we have $\psi(z) \rightarrow \infty$ if and only if $z \rightarrow \infty$. Then put

$$
v_{n}(z)=\log \left|\psi\left(f^{n}(z)\right)\right|, \quad z \in \bar{U}, n \in \mathbb{N} .
$$

Each $v_{n}$ is positive harmonic in $U$ and continuous in $\bar{U}$, and we find by using Egorov's theorem again that there exists a closed set $E \subset \partial U$ such that $\omega(z, E, U)>$ 0 for $z \in U$ and

$$
v_{n} \rightarrow \infty \text { as } n \rightarrow \infty, \quad \text { uniformly on } E \text {. }
$$

As in the first case, we can use Lemma 3.1 to deduce that

$$
v_{n}(z)=\log \left|\psi\left(f^{n}(z)\right)\right| \rightarrow \infty \text { as } n \rightarrow \infty, \quad \text { for } z \in U .
$$

Hence $f^{n}(z) \rightarrow \infty$ as $n \rightarrow \infty$ for $z \in U$, as required.

Part (b). This proof is similar to that of part (a). Suppose that $\partial U \cap A(f)$ has positive harmonic measure relative to $U$. By the definition of $A(f)$, we have

$$
A(f)=\bigcup_{L \in \mathbb{N}} A_{R}^{-L}(f),
$$


where $R>1$ is so large that $R_{n}=M^{n}(R) \rightarrow \infty$ as $n \rightarrow \infty$ and

$$
A_{R}^{-L}(f)=\left\{z:\left|f^{n+L}(z)\right| \geq R_{n}, \text { for } n \in \mathbb{N}\right\}, \quad L \in \mathbb{N} .
$$

Thus $\partial U \cap A_{R}^{-L}(f)$ has positive harmonic measure relative to $U$ for some $L \in \mathbb{N}$. Hence there exists a closed set $E \subset \partial U$ of positive harmonic measure relative to $U$ such that

$$
\left|f^{n+L}(\zeta)\right| \geq R_{n}, \quad \text { for } \zeta \in E, n \in \mathbb{N} .
$$

As in the proof of part (a), in the ' $F(f)$ disconnected' case, we deduce by Lemma 3.1 that

$$
\log \left|f^{n+L}(z)\right| \geq\left(\log R_{n}\right) \omega(z, E, U), \quad \text { for } z \in U, n \in \mathbb{N},
$$

so

$$
\left|f^{n+L}(z)\right| \geq R_{n}^{\omega(z, E, U)}, \quad \text { for } z \in U, n \in \mathbb{N} .
$$

For any fixed $z \in U$, we can choose $N$ so large that

$$
R_{n}^{\omega(z, E, U)} \geq R_{n-1}, \quad \text { for } n \geq N,
$$

because $\log M(r) / \log r \rightarrow \infty$ as $r \rightarrow \infty$. So for this $z$ we have

$$
\left|f^{n+L}(z)\right| \geq R_{n-1}, \quad \text { for } n \geq N,
$$

and, moreover,

$$
\left|f^{n+L+1}(z)\right| \geq R_{n}, \quad \text { for } n \in \mathbb{N},
$$

because $|f(z)| \geq M(r)$ implies that $|z| \geq r$ for $r>0$. Thus $z \in A_{R}^{-L-1}(f)$.

The proof in the ' $F(f)$ connected' case is similar, using the fact that, for $|z|$ large enough, the function $\psi$ defined in (3.2) satisfies

$$
\frac{k}{2}|z|^{1 / 2} \leq|\psi(z)| \leq 2 k|z|^{1 / 2}
$$

It follows from (3.3) and (3.4) that, for some $N \in \mathbb{N}$, we have

$$
\left|\psi\left(f^{n+L}(\zeta)\right)\right| \geq(k / 2) R_{n}^{1 / 2}, \quad \text { for } \zeta \in E, n \geq N,
$$

so, using Lemma 3.1 as before, we deduce that

$$
\log \left|\psi\left(f^{n+L}(z)\right)\right| \geq\left(\log \left((k / 2) R_{n}^{1 / 2}\right)\right) \omega(z, E, U), \quad \text { for } z \in U, n \geq N .
$$

Hence

$$
\left|\psi\left(f^{n+L}(z)\right)\right| \geq\left((k / 2) R_{n}^{1 / 2}\right)^{\omega(z, E, U)}, \quad \text { for } z \in U, n \geq N,
$$

so, by (3.4) again, there exists $c>0$ such that

$$
\left|f^{n+L}(z)\right| \geq c R_{n}^{\omega(z, E, U)}, \quad \text { for } z \in U, n \geq N .
$$

The rest of the proof is similar to that of the ' $F(f)$ connected' case in part (a).

Remarks. 1. If the Fatou component $U$ is bounded, then the proofs of both parts of Theorem 1.2 can be simplified considerably since only the ' $F(f)$ disconnected' case can occur, and Lemma 3.1 can be replaced by the ordinary maximum principle.

2. In Theorem 1.2, part (a), the condition that $\partial U \cap I(f)$ has Hausdorff dimension 2 would not imply that $U \subset I(f)$. For example, functions of the form $f(z)=\lambda e^{z}, 0<\lambda<1 / e$, have a completely invariant attracting basin $U$ and the Hausdorff dimension of $\partial U \cap I(f)=J(f) \cap I(f)$ is 2; see [11]. 


\section{Some consequences of Theorems 1.1 and 1.2}

In this section we prove two results about $I(f)$ which follow from Theorems 1.1 and 1.2, respectively. We thank Lasse Rempe for pointing out that part (b) of Theorem 4.1 can be stated in the more interesting way given in part (c).

Theorem 4.1. Let $f$ be a transcendental entire function. The following statements hold.

(a) Any bounded component of $I(f)$ meets $J(f)$.

(b) If $G$ is a bounded simply connected domain and $G \cap I(f) \neq \emptyset$, then $\partial G \cap$ $I(f) \neq \emptyset$.

(c) $I(f) \cup\{\infty\}$ is connected.

To prove Theorem 4.1 we need a lemma whose proof is based on the blowing up property of $J(f)$ :

if $f$ is an entire function, $K$ is compact, $K \subset \mathbb{C} \backslash E(f)$ and $V$ is an open neighbourhood of $z \in J(f)$, then there exists $N \in \mathbb{N}$ such that $f^{n}(V) \supset K$, for all $n \geq N$.

Here $E(f)$ is the exceptional set of $f$, that is, the set of points with a finite backwards orbit under $f$, which has at most one point (see [3]).

Lemma 4.1. Let $f$ be a transcendental entire function. If $G$ is a bounded simply connected domain such that $G \cap J(f) \neq \emptyset$, then $\partial G \cap I(f) \neq \emptyset$.

Proof. Since $G$ is a bounded domain, we can define $\alpha_{n}, n \in \mathbb{N}$, to be the outer boundary component of $f^{n}(G)$. Then, by the blowing up property of $J(f)$, we have $\operatorname{dist}\left(\alpha_{n}, 0\right) \rightarrow \infty$ as $n \rightarrow \infty$. The compact sets

$$
K_{n}=\left\{z \in \partial G: f^{n}(z) \in \alpha_{n}\right\}, \quad n \in \mathbb{N},
$$

form a nested sequence, since $\alpha_{n+1} \subset f\left(\alpha_{n}\right)$, for $n \in \mathbb{N}$, so $K=\bigcap_{n \geq 1} K_{n} \neq \emptyset$. All points of $K$ must lie in $I(f)$, so $\partial G \cap I(f) \neq \emptyset$, as required.

Proof of Theorem 4.1. Part (a) follows immediately from Theorem 1.1. For if $I_{0}$ is a bounded component of $I(f)$ that does not meet $J(f)$, then $I_{0}$ must be a bounded Fatou component, and this contradicts the fact that such a component must have a boundary point in $I(f)$, by Theorem 1.1.

To prove part (b) we suppose that $G$ is a bounded simply connected domain that meets $I(f)$. By Lemma 4.1. we can assume that $G \subset F(f)$, so $G \subset U$, where $U$ is an escaping Fatou component of $f$. There are now two cases:

- either $\partial G \cap U \neq \emptyset$, in which case $\partial G \cap I(f) \neq \emptyset$;

- or $G=U$, in which case $U$ is an escaping wandering domain, so $\partial G \cap I(f) \neq$ $\emptyset$, by Theorem 1.1 .

To prove part (c) we note that if $I(f) \cup\{\infty\}$ is disconnected, then there exist disjoint open sets $H_{1}$ and $H_{2}$ in the Riemann sphere $\hat{\mathbb{C}}$ such that

$$
I(f) \cup\{\infty\} \subset H_{1} \cup H_{2} \quad \text { and } \quad H_{i} \cap(I(f) \cup\{\infty\}) \neq \emptyset \text {, for } i=1,2 .
$$

Without loss of generality, $\infty \in H_{2}$ and $H_{1}$ is bounded. Since $I(f)$ meets $H_{1}$ it also meets $\partial H_{1}$ by part (b), and this gives a contradiction.

(Part (c) clearly implies part (b), so these two statements are equivalent.) 
Remarks. 1. The proof of Lemma 4.1 given here is a variation of the proof by Domínguez [6] that $I(f) \neq \emptyset$, in which the use of Bohr's theorem is replaced by the use of the blowing up property of $J(f)$.

2. An alternative proof of Lemma 4.1 can be given, based on the following properties of the fast escaping set $A(f)$ (see [5] and [14):

$$
\partial A(f)=J(f)
$$

all the components of $A(f)$ are unbounded.

Since $G \cap J(f) \neq \emptyset$, we have $G \cap A(f) \neq \emptyset$, by (4.1). Hence $\partial G \cap A(f) \neq \emptyset$, by (4.2), which proves Lemma 4.1. In fact, the set $K$ constructed in the proof of Lemma 4.1] is a subset of $A(f)$ by [17, Corollary 2.5].

3. There is also a constructive proof of Theorem 4.1, part (c). First prove Theorem 4.1, part (a) as above. Then consider $A_{0}=A(f) \cup\{\infty\}$. By (4.2), $A_{0}$ is a union of connected sets in $\hat{\mathbb{C}}$, all containing $\infty$, so $A_{0}$ is connected. Let $I_{0}$ be the component of $I(f) \cup\{\infty\}$ that contains $A_{0}$. Any point of $I(f)$ either lies in an unbounded component of $I(f)$, and so is in $I_{0}$, or it lies in a bounded component of $I(f)$, which must contain a point of $J(f)$, by Theorem 4.1, part (a). By (4.1), such a component of $I(f)$ must also be in $I_{0}$. Hence $I(f) \cup\{\infty\}=I_{0}$.

Next we use Theorem 1.2 to give a sufficient condition for $I(f)$ to be connected.

Theorem 4.2. Let $f$ be a transcendental entire function and suppose that there exists a bounded domain $G$ such that

$$
\partial G \subset I(f) \text { and } \quad G \cap I(f)^{c} \neq \emptyset .
$$

Then

(a) for each $n \in \mathbb{N}$, the outer boundary component $\alpha_{n}$ of $f^{n}(G)$ is contained in $I(f), \alpha_{n} \rightarrow \infty$ as $n \rightarrow \infty$, and $\alpha_{n}$ surrounds 0 for sufficiently large $n$;

(b) $I(f)$ is connected.

The statement of Theorem 4.2 is similar to that of [15, Theorem 2], but here we assume that $G$ satisfies (4.3), whereas in [15, Theorem 2] we assumed that

$$
\partial G \subset I(f) \text { and } G \cap J(f) \neq \emptyset .
$$

In [15], we remarked that (4.3) and (4.4) are equivalent, but we later realised that the statement that (4.3) implies (4.4) is not immediate. The following lemma shows that this implication can be deduced from Theorem 1.2 .

Lemma 4.2. Let $f$ be a transcendental entire function. Then the conditions (4.3) and (4.4) are equivalent.

Proof. It is clear that if $G \cap J(f) \neq \emptyset$, then $G \cap I(f)^{c} \neq \emptyset$, since $J(f)=\partial I(f)$, by (1.1). Thus (4.4) implies (4.3).

To prove that (4.3) implies (4.4) we argue as follows. If $\partial G \subset I(f)$ and $G \subset F(f)$, then either $\partial G \cap F(f) \neq \emptyset$, in which case $G \subset I(f)$ or $G$ is a component of the Fatou set, so $G \subset I(f)$ by Theorem 1.2, part (a). Hence (4.3) implies (4.4).

Theorem 4.2 follows immediately from Lemma 4.2 and [15, Theorem 2]. However, the proof below contains a shorter argument to prove part (b) than that given in [15]. (In [15] a constructive argument was used, whereas here we argue by contradiction.) 
Proof of Theorem 4.2, By Lemma 4.2, the hypothesis (4.3) implies that $G \cap J(f) \neq$ $\emptyset$.

Since $\partial G \subset I(f)$, the outer boundary component $\alpha_{n}$ of $f^{n}(G)$ is contained in $I(f)$, for each $n \in \mathbb{N}$. By the blowing up property of $J(f), \alpha_{n} \rightarrow \infty$ as $n \rightarrow \infty$, and $\alpha_{n}$ surrounds 0 for sufficiently large $n$. Thus part (a) holds.

To prove part (b) suppose that $I(f)$ is disconnected. Then there exist disjoint open sets $H_{1}$ and $H_{2}$ in $\mathbb{C}$ such that

$$
I(f) \subset H_{1} \cup H_{2} \quad \text { and } \quad H_{i} \cap I(f) \neq \emptyset \text {, for } i=1,2 .
$$

Without loss of generality, one of $H_{1}$ and $H_{2}$ is bounded, say $H_{1}$, for otherwise both $H_{1}$ and $H_{2}$ have unbounded boundary components that do not meet $I(f)$, which contradicts part (a). We can also assume that $H_{1}$ is simply connected.

Fix $z_{0} \in H_{1} \cap I(f)$ and take any $m \in \mathbb{N}$. Then there exists $N_{m} \in \mathbb{N}$ such that

$$
f^{n}\left(z_{0}\right) \text { lies outside } \alpha_{m}, \quad \text { for } n \geq N_{m} .
$$

Now, for $n \in \mathbb{N}$,

$$
\partial f^{n}\left(H_{1}\right) \subset f^{n}\left(\partial H_{1}\right) \subset I(f)^{c},
$$

because $\partial H_{1} \subset I(f)^{c}$. Also, $f^{n}\left(\partial H_{1}\right)$ is connected because $\partial H_{1}$ is connected. Thus, since $\alpha_{m} \subset I(f)$, it follows from (4.5) and (4.6) that

$$
f^{n}\left(\partial H_{1}\right) \text { lies outside } \alpha_{m}, \quad \text { for } n \geq N_{m} .
$$

Thus $f^{n}\left(\partial H_{1}\right) \rightarrow \infty$ as $n \rightarrow \infty$, so $\partial H_{1} \subset I(f)$, a contradiction. Hence $I(f)$ is connected.

Remarks. 1. The final part of this contradiction argument can be replaced by an argument based on the use of Lemma 4.1 and Theorem 4.1.

2. In Theorem 4.2 the set $I(f)$ has the structure of a 'spider's web', a concept introduced in [17]: we say that a set $E$ is an (infinite) spider's web if $E$ is connected and there exists a sequence $\left(G_{n}\right)$ of bounded simply connected domains with $G_{n} \subset$ $G_{n+1}, \partial G_{n} \subset E$, for $n \in \mathbb{N}$, and

$$
\bigcup_{n=1}^{\infty} G_{n}=\mathbb{C}
$$

Several examples of functions for which $I(f)$ is a spider's web, and hence $I(f)$ is connected, were given in [15, Section 6], though the name 'spider's web' was not used there. Many more examples of such functions are given in [17] and [18.

Condition (4.3) can be described by saying that $I(f)$ has a hole, so Theorem 4.2 can be stated in the following geometric way:

$$
\text { if } I(f) \text { has a hole, then } I(f) \text { is a spider's web. }
$$

3. The proof of Theorem 4.2 given above was inspired by unpublished work of Professor Noel Baker. In early 2010 the authors found a short note by Noel Baker written (some time after 1996) in an old diary that he used for rough work, which included the statement: 'I think I can show that for the examples of entire functions with 'rings' the set $I(f)$ is connected.' He did not give details of the proof, but the note suggested that it was by contradiction and used the method of showing that $I(f) \neq \emptyset$ given by Domínguez in [6].

Noel Baker died in 2001 and as far as we know he did not mention this result to anyone. In 14 we proved the result as a corollary of the fact that all the 
components of $A(f)$ are unbounded. Thinking about the argument he might have found led us to the proof of Theorem 4.2 above.

\section{Components of $I(f)$}

In this final section, we give various results about the possible structures of the components of $I(f)$. The first result, which follows from Theorem 1.1 gives a new sufficient condition for all the components of $I(f)$ to be unbounded.

Theorem 5.1. Let $f$ be a transcendental entire function and $E$ be a set such that $J(f) \subset \bar{E}$. If $E$ is contained in the union of finitely many components of $I(f)$, then

(a) $I(f) \cap J(f)$ is contained in one component, $I_{1}$ say, of $I(f)$;

(b) all the components of $I(f)$ are unbounded, and they consist of

(i) $I_{1}$, which also contains any escaping wandering domains and any Baker domains of $f$ with at least one boundary point in $I(f)$,

(ii) any Baker domains of $f$ with no boundary points in $I(f)$, and the infinitely many preimage components of such Baker domains.

Remark. If every Baker domain of $f$ has a point of $I(f)$ on its boundary, then the conclusion of Theorem 5.1 can be strengthened to ' $I(f)$ is connected'.

Proof of Theorem 5.1. Suppose that $E$ is contained in the union of finitely many components of $I(f)$, say $I_{1}, I_{2}, \ldots, I_{m}$. Take any $z \in I(f) \cap J(f)$. Since $J(f) \subset \bar{E}$, there exist $z_{n} \in E$ such that $z_{n} \rightarrow z$ as $n \rightarrow \infty$. Without loss of generality all terms of this sequence $\left(z_{n}\right)$ lie in a single component, $I_{j}$ say. Since $z \in I(f)$, we have $z \in I_{j}$. Hence

$$
I(f) \cap J(f) \subset I_{1} \cup I_{2} \cup \cdots \cup I_{m} .
$$

We now assume that $I_{1}, I_{2}, \ldots, I_{m}$ is the minimal set of components of $I(f)$ such that (5.1) holds. Then $I_{j} \cap J(f) \neq \emptyset$, for $j=1,2, \ldots, m$. Note that if the exceptional set $E(f)$ is non-empty, then

$$
\left(I_{j} \backslash E(f)\right) \cap J(f) \neq \emptyset, \quad \text { for } j=1,2, \ldots, m .
$$

Indeed, if $E(f)=\{\alpha\} \subset I_{j} \cap J(f)$, then $\alpha$ is a limit point of the backwards orbit of any non-exceptional point in $I(f) \cap J(f)$ (this follows from the blowing up property of $J(f))$ and hence $\alpha$ is the limit of a sequence in $I_{i} \cap J(f)$, say, by (5.1). Thus $i=j$ and so (5.2) holds.

If $m=1$, then $I(f) \cap J(f)$ is contained in one component of $I(f)$, as required. If $m>1$, then we can take $z_{1} \in I_{1} \cap J(f)$ and an open disc $D$ centred at $z_{1}$ so small that

$$
D \cap\left(I_{2} \cup \cdots \cup I_{m}\right)=\emptyset .
$$

Consider $I_{j}, j \geq 2$. Then there exists $N \in \mathbb{N}$ such that $f^{N}(D)$ meets both $I_{1} \cap J(f)$ and $I_{j} \cap J(f)$, by (5.2) and the blowing up property. Hence there exist $w_{1}, w_{j} \in D$ such that

$$
f^{N}\left(w_{1}\right) \in I_{1} \cap J(f) \quad \text { and } \quad f^{N}\left(w_{j}\right) \in I_{j} \cap J(f),
$$

so $w_{1}, w_{j} \in I_{1}$ by the backwards invariance of $I(f) \cap J(f)$ and (5.3). Thus $f^{N}\left(I_{1}\right)$ is a connected subset of $I(f)$ that meets both $I_{1}$ and $I_{j}$, which is a contradiction. Hence $m=1$, so part (a) holds.

Clearly the component $I_{1}$ is unbounded. If $U$ is a wandering domain in $I(f)$, then $\partial U \cap I(f) \neq \emptyset$ by Theorem 1.1 so $\partial U \cap I_{1} \neq \emptyset$ and hence $U \subset I_{1}$. Similarly, if 
$U$ is a Baker domain whose boundary meets $I(f)$, then $U \subset I_{1}$; in particular, this is true if $U$ is completely invariant, since in this case $\partial U=J(f)$.

To complete the proof of part (b) we note that any other component of $I(f)$ must be a Baker domain that has no points of $I(f)$ in its boundary, and so is not completely invariant, or it must be one of its infinitely many preimage Fatou components, all of which are unbounded.

Theorem 5.1 includes the following special cases.

Corollary 5.1. Let $f$ be a transcendental entire function.

(a) Either $I(f)$ is connected or it has infinitely many components.

(b) If $A(f)$ is contained in the union of finitely many components of $I(f)$, then all the components of $I(f)$ are unbounded.

In part (b) the set $A(f)$ can be replaced by any subset of $I(f)$ whose closure contains $J(f)$. Such subsets of $I(f)$ include the sets $L(f), M(f)$ and $Z(f)$, all of which are defined in [16].

The proof of Theorem 5.1, and hence of Corollary 5.1, used Theorem 1.1 together with the blowing up property of $J(f)$. In fact, we can use just the blowing up property to prove part (a) of Corollary 5.1. More generally, we have the following result which is not directly related to escaping points.

Theorem 5.2. Let $f$ be a transcendental entire function and suppose that the set $E$ is completely invariant under $f$ and that $J(f)=\overline{E \cap J(f)}$. Then exactly one of the following holds:

(1) E is connected;

(2) E has exactly two components, one of which is a singleton $\{\alpha\}$, where $\alpha$ is a fixed point of $f$ and $\alpha \in E(f) \cap F(f)$;

(3) E has infinitely many components.

Proof. We can assume that $E$ is infinite, since any completely invariant finite set must be a singleton.

Suppose that cases (1) and (3) do not hold. Then $E$ has finitely many components $E_{1}, E_{2}, \ldots, E_{m}$, say, with $m>1$. Since $J(f)=\overline{E \cap J(f)}$, we can assume that $E_{1} \neq E(f)$ and that there exists $z_{1} \in\left(E_{1} \backslash E(f)\right) \cap J(f)$. Now suppose that, for some $j \geq 2$, we have $E_{j} \neq E(f)$. Choose an open disc $D$ centred at $z_{1}$ and not meeting $E_{2} \cup \cdots \cup E_{m}$. For some $N \in \mathbb{N}, f^{N}(D)$ meets both $E_{1}$ and $E_{j}$, by the blowing up property, so $f^{N}\left(E_{1}\right)$ meets both $E_{1}$ and $E_{j}$ by the backwards invariance of $E$. But $f^{N}\left(E_{1}\right)$ is a connected subset of $E$, by the forwards invariance of $E$, which is a contradiction.

Thus $E$ has two components $E_{1}$ and $E(f)=\{\alpha\}$, say. Then $\alpha \in F(f)$, since any point in $E \cap J(f)$ lies in $E_{1}$ because it is a limit point of the backwards orbit of $z_{1}$ (this backwards orbit lies in $E$ by the backwards invariance of $E$ ).

Finally we show that $f(\alpha)=\alpha$. If $f(\alpha) \neq \alpha$, then $f(\alpha) \in E_{1}$, by the complete invariance of $E$. Since $E_{1}$ is infinite, $f(\alpha)$ is a limit point of $E$ and so therefore is $\alpha$, by the complete invariance of $E$ and the local behaviour of $f$ near $\alpha$. Thus we have a contradiction. Hence $\alpha$ is a fixed point of $f$ and case (2) holds.

Remarks. 1. The function $f(z)=\frac{1}{2} z^{2} e^{2-z}$ shows that case (2) of Theorem 5.2 can occur, with $E=J(f) \cup\{0\}$. In this case $J(f)$ is connected and 0 is a fixed point of $f$ in $E(f) \cap F(f)$ (see [10, proof of Theorem 4]). 
2. Taking $E$ to be the grand orbit of a non-exceptional point in $J(f)$ shows that in case (3) of Theorem 5.2 the set $E$ may have only countably many components.

Note that case (2) of Theorem 5.2 cannot occur if we also know, for example, that $E \subset I(f)$ or that $E$ contains a neighbourhood of every fixed point of $f$ in $F(f)$. Thus if $E$ is $I(f), L(f), M(f), Z(f)$ or $A(f)$, or the complement of one of these sets, then $E$ is either connected or has infinitely many components.

It is known that if $f$ is a transcendental entire function, then $J(f)$ is either connected or it has uncountably many components (see [2, Theorem B]), and it is natural to ask if $I(f)$ and $A(f)$ also have this property.

In Theorem 5.2 the case when $E$ has infinitely many components can be strengthened to state that each neighbourhood of each point $z_{0} \in J(f)$ must meet infinitely many components of $E$. This is implied by the following simple result.

Theorem 5.3. Let $A$ and $B$ be completely invariant sets for a transcendental entire function $f$. If, for some $z_{0} \in J(f)$, there is a neighbourhood $D$ of $z_{0}$ such that $A \cap D$ meets only finitely many components of $B$, then $A$ meets only finitely many components of $B$.

Proof. Suppose that $A \cap D \subset B_{1} \cup \cdots \cup B_{m}$, a minimal union of components of $B$. Let $K$ be any compact set, not meeting the exceptional set $E(f)$. Then, by the blowing up property of $J(f)$, there exists $N \in \mathbb{N}$ such that $f^{N}(D) \supset K$. Thus $A \cap K$ meets at most $m$ components of $B$, by the complete invariance of $A \cap B$. Hence $A$ meets exactly $m$ components of $B$ in $\mathbb{C} \backslash E(f)$, namely $B_{1}, \ldots, B_{m}$, and so $A$ meets at most $m+1$ components of $B$ in $\mathbb{C}$.

By applying Theorem 5.3 with $A=\mathbb{C}$ and $B=E$, we deduce that if $E$ is completely invariant and has infinitely many components, then each neighbourhood of each point $z_{0} \in J(f)$ must meet infinitely many components of $E$; in particular, this is the case in Theorem 5.2 , case (3).

Other choices of the sets $A$ and $B$ in Theorem 5.3 give other corollaries. For example, with $A=A(f)$ and $B=I(f)$, Theorem 5.3 and Corollary 5.1, part (b), combine to give the following result.

Corollary 5.2. Let $f$ be a transcendental entire function. If, for some $z_{0} \in J(f)$, there is a neighbourhood $D$ of $z_{0}$ such that $A(f) \cap D$ is contained in the union of finitely many components of $I(f)$, then $A(f)$ is contained in the union of finitely many components of $I(f)$, so all the components of $I(f)$ are unbounded.

\section{ACKNOWLEDGEMENTS}

The authors thank Walter Bergweiler, Dan Nicks and Dave Sixsmith for their useful comments; Tim Stallard for drawing the figure; and Lasse Rempe for a very nice observation about the statement of Theorem 4.1

\section{REFERENCES}

[1] I. N. Baker, Completely invariant domains of entire functions, Mathematical Essays Dedicated to A. J. Macintyre, 33-35, Ohio Univ. Press, Ohio, 1970. MR0271344 (42:6227)

[2] I. N. Baker and P. Domínguez, Some connectedness properties of Julia sets, Complex variables, 41 (2000), 371-389. MR1785150(2001e:30039)

[3] W. Bergweiler, Iteration of meromorphic functions, Bull. Amer. Math. Soc., 29 (1993), 151188. MR 1216719 (94c:30033)

[4] W. Bergweiler, An entire function with simply and multiply connected wandering domains, Pure Appl. Math. Quarterly, 7 (2011), 107-120. 
[5] W. Bergweiler and A. Hinkkanen, On semiconjugation of entire functions, Math. Proc. Cambridge Philos. Soc., 126 (1999), 565-574. MR.1684251 (2000c:37057)

[6] P. Domínguez, Dynamics of transcendental meromorphic functions, Ann. Acad. Sci. Fenn. Math., 23 (1998), 225-250. MR1601879 (99b:30031)

[7] A. E. Eremenko, On the iteration of entire functions, Dynamical systems and ergodic theory, Banach Center Publications, 23, Polish Scientific Publishers, Warsaw, 339-345, 1989. MR.1102727 (92c:30027)

[8] P. Fatou, Sur l'itération des fonctions transcendantes entières, Acta Math., 47 (1926), 337370. MR.1555220

[9] J. B. Garnett and D. E. Marshall, Harmonic measure, Cambridge University Press, 2005. MR2150803 (2006g:31002)

[10] M. Kisaka, On the connectivity of Julia sets of entire functions, Ergodic Theory and Dynam. Systems, 18 (1998), 189-205. MR1609471 (99a:30033)

[11] C. McMullen, Area and Hausdorff dimension of Julia sets of entire functions, Trans. Amer. Math. Soc., 300 (1987), 329-342. MR871679 (88a:30057)

[12] T. Ransford, Potential theory in the complex plane, Cambridge University Press, 1995. MR1334766 (96e:31001)

[13] P. J. Rippon, Baker domains, Transcendental dynamics and complex analysis, Cambridge University Press, 371-395, 2008. MR2458809 (2009j:30061)

[14] P. J. Rippon and G. M. Stallard, On questions of Fatou and Eremenko, Proc. Amer. Math. Soc., 133 (2005), 1119-1126. MR2117213 (2005j:37069)

[15] P. J. Rippon and G. M. Stallard, Escaping points of entire functions of small growth, Math. Z., 261 (2009), 557-570. MR2471088 (2010a:30043)

[16] P. J. Rippon and G. M. Stallard, Slow escaping points of meromorphic functions, to appear in Trans. Amer. Math. Soc., arXiv: 0812.2410.

[17] P. J. Rippon and G. M. Stallard, Fast escaping points of entire functions, arXiv: 1009.5081.

[18] D. J. Sixsmith, Entire functions for which the escaping set is a spider's web, arXiv: 1012.1303.

[19] W. Rudin, Real and complex analysis, McGraw-Hill, 1966. MR0210528 (35:1420)

Department of Mathematics and Statistics, The Open University, Walton Hall, Milton Keynes MK7 6AA, United Kingdom

E-mail address: p.j.rippon@open.ac.uk

Department of Mathematics and Statistics, The Open University, Walton Hall, Milton Keynes MK7 6AA, United Kingdom

E-mail address: g.m.stallard@open.ac.uk 